Aus der Hoffaschen Klinik in Berlin.

\section{Pathologisch-anatomische Beiträge zur Lehre des chronischen Gelenkrheumatismus und der Arthritis deformans.')}

\section{Von Dr. G. A. Wollenberg.}

Die Beobachtung, daß das Röntgenbild bei der primär chronischen progressiven Polyarthritis Deformationen im engeren Sinne, d. h. neben Knorpel- und Knochenschwund auch Knorpel- und Knochenwucherungen, in der Regel nicht erkennen läßt, wird durch unsere pathologisch-anatomischen Untersuchungen bestätigt. Das Röntgenbild gibt naturgemäß keine Aufschlüsse über die feineren Veränderungen im Knorpel und Knochen. Von den bisher in der Literatur vorliegenden Berichten über die Anatomie der progessiven Polyarthritis ist ein großer Teil nicht zu verwerten, da viele Autoren die Krankheitsbilder der primär chronischen progessiven Polyarthritis und des sekundär chronischen Gelenkrheumatismus einerseits, das der Arthritis deformans anderseits nicht streng auseinander hielten, vielmehr die Anatomie der letzteren Erkrankung einfach den beiden erstgenannten Krankheitsgruppen unterschoben. Die meisten der genau klassifizicrten und genau anatomisch studierten Fälle der chronischen Polyarthritiden stellen Endstadien vor, aus denen man natürlich auch nur wenig Schlüsse auf die Anfangsstadien ziehen kann. Hierzu gehören die von B on net, Waldmann, Janssen, Kachel u. a. beschriebenen Fälle, während Frühstadien bisher fast nur im Kindesalter anatomisch untersuch wurden (Delcourt, Johannessen, Spitzy). Nur Schüller hat auch bei Erwachsenen frühe Stadien des anatomischen Prozesses gesehen und beschrieben. Unsere Untersuchungen hatten sich nun die Aufgabe gestellt, sowohl bei der Arthritis deformans, als auch bei den chronischen Polyarthritiden womöglich die Entwicklung der Läsionen an der Synovialis einerseits, am Knorpel und Knochen anderseits zu studieren und so das gegenseitige Verhältnis dieser Läsionen zueinander klar zu legen.

Die Resultate unserer makro- und mikroskopischen Untersuchungen sind nun folgende:

\section{Arthritis deformans (im Sinne Volkmanns):}

Der Krankheitsprozeß äußert sich zuerstim Knorpel und Knochen, während die Synovialis anfangs nur ganz unbedeutende Veränderungen aufweist. Wenn die Auffaserung und die Proliferationserscheinungen am Knorpel schon sehr ausgesprochen sind, sehen wir an der Synovialis nur eine einfache Zottenhyperplasie mit geringer Gefäß- und Bindegewebsneubildung. Erst wenn die knorpeligen und knöchernen Gelenkbestandteile hochgradig verändert sind, wenn ausgedehnte Usuren und Schlifflächen, drusige Randwucherungen vorhanden sind, wurden auch die eben geschilderten Veränderungen an der Synovialis stärker ausgeprägt. Stets aber sind die enzündlichen Erscheinungen an der Synovialmembran nur gering; es kommen nur selten spärliche, kleine Lymphocytenanhäufungen vor, nie aber auch nur in annähernder Weise, wie bereits in den frühen Stadien der primär chronischen Polyarthritis. $\mathrm{Da}$ gegen treten in den späteren Stadien der Arthritis deformans degenerative Veränderungen der Synovialis, speziell die hyaline Degeneration mehr in den Vordergrund. So kann die ganze Synovialis in ein strukturloses, hyalines Gewebe verwandelt werden.

Ferner finden sich Verkalkungen mit Pigmenteinlagerungen (intra- und extrazellulär) oft sehr reichlich.

Wenn wir diese Veränderungen mit anderen krankhaften Prozessen der Synovialis vergleichen, so erinnern dieselben am meisten an die anatomischen Bilder der traumatischen Synovialiserkrankung, die wir in großer Anzahl zu untersuchen Gelegenheit hatten, und wir sind daher geneigt, einen großen Teil der anatomischen Synovialisveränderungen bei der Arthritis deformans auf das „innere Trauma“ zurückzuführen, dem die Synovialmembran bei jeder Bewegung der deformierten Gelenke ausgesetzt ist.

2. Primär chronische progressive Polyarthritis.

Der krankhafte Prozeß beginnt hier im strikten Gegensatz zur Arthritis deformans in der Synovialis, während der Knorpel zunächst keine Veränderungen aufweist. In frühen Stadien der Erkrankung sehen wir eine viel stärkere Zottenwucherung der Synovialis, viel reichere Gefäßneubildung, viel bedeutendere Proliferation der fixen Bindegewebszellen, als wir dies sogar in den viel weiter fortgeschrittenen Stadien der Arthritis deformans je antreffen; das Charakteristischste aber sind mächtige, meist dicht beieinander gelegene Anhäufungen von Rundzellen, die meist zirkumskripte, p erivasculäre, lymphomartige Gebilde darstellen; daneben finden wir auch hier und da eine diffuse Durchsetzung des Gewebes mit Lymphocyten.

Diese lymphomartigen Rundzellenanhäufungen findet man auch bei verschiedenen anderen Erkrankungen der Gelenke, sie sind dort jedoch fast nie so enorm zahlreich und dicht; natürlich stellen sie nichts Spezifisches dar, und speziell unterscheiden sie sich vom Tuberkelknötchen ganz scharf. Veränderungen des Knorpels und Knochens in diesen frühen Stadien sind entweder noch garnicht vorhanden, oder rein regressiver Natur, indem die Knorpel der Usur verfallen, Erosionen aufweisen, oder aber von einem der Synovialis entstammenden pannusartigen Granulationsgewebe substituiert werden. Dadurch wird die Knorpelfläche allmählich in Bindegewebe verwandelt.

In späteren Stadien können dann auch scheinbar geringe Proliferationserscheinungen auftreten, und zwar besonders in milde verlaufenden Fällen. So kann es unter dem Einflusse des den Knorpel überwuchernden Granulationsgewebes zu einer geringen Aufquellung der Knorpelgrundsubstanz kommen. Wirkliche Proliferation von Knorpelzellen konnten wir aber nur in sehr geringem Grade feststellen. Es kommt am Rande des Knorpels zu einem Durchbruch der Corticalis, zu einer Einwucherung von Granulationsgewebe, das ebenfalls von der Synovialis stammt, in die Markhöhle.

In ganz alten Stadien der Affektion finden wir schließlich eine starke bindegewebige Verdickung und Schrumpfung der Gelenkkapsel, ferner mehr oder weniger totale Verwachsung der gegenüberliegenden Synovialispartien und der Gelenkenden. Ueberall in der Nähe der Verwachsungsflächen aber finden wir wieder mehr oder weniger mächtige Lymphocytenanhäufungen und -infiltrationen. Der Knorpel ist nun ganz oder fast ganz verschwunden, resp. durch Bindegewebe ersetzt, sodaß oft, da auch die Corticalis des Knochens stark zerstört ist, nur eine dünne Bindegewebslamelle die Begrenzung der Markhöhle bildet. Die knöchernen Gelenkenden sind stark verkleinert, ihre Spongiosa äußerst rarefiziert, in spärliche, sehr dünne Bälkchen verwandelt. Knorpel- und Knochenwucherungen haben wir nicht konstatieren können.

Vergleichen wir diese anatomischen Veränderungen mit denen bei anderen Gelenkerkrankungen, so ergibt sich eine große Aehnlichkeit der Synovialisläsionen mit denen bei infektiösen Arthritiden bekannter Aetiologie, z. B. mit der gonorrhoischen Arthritis.

3. Sekundär chronischer Gelenkrheumatismus. Die Veränderungen sind denen bei primär chronischer progressive Polyarthritis durchaus analog, und haben wir bei unseren Untersnchungen prinzipielle Unterschiede nicht auffinden können; speziell scheint uns die fibröse Verwachsung nichts für die sekundär chronischen Polyarthritiden Charakteristisches zu sein (gegenüber $\mathrm{J}$ accoud)

Die von. uns angestellten Untersuchungen beweisen demnach, daß die Arthritis deformans und die beiden von uns studierten Formen der chronischen progressiven Polyarthritis sich auch anatomisch scharf voneinander unterscheiden, und sowohl in ihren frühen, wie in ihren späten Stadien. Wir glauben daher, daß das eventuelle Auftreten deformierender Prozesse bei eine dieser beiden chronischen progressiven Polyarthritiden lediglich eine sekundäre Komplikation, die Reaktion des Knorpels und Knochens auf die durch die Synovitis bedingte Ernährungsstörung darstellt. 